\title{
ABOUT THE GIBBS-APPEL EQUATIONS FOR MULTIBODY SYSTEMS
}

\author{
NGUYEN VAN KHANG \\ Department of Applied Mechanics, Hanoi University of Technology
}

\begin{abstract}
In this paper a matrix form of Gibbs-Appel function is recommended for multibody dynamics formulations. The form proposed in this paper seems to be more clear and suitable for automatic generation of dynamical equations of motion. The advantages followed from the formulation proposed are illustrated through an example.
\end{abstract}

\section{INTRODUCTION}

Gibb-Appel equations were introduced by Gibbs in 1879 [1] and were subsequently studied and formalized by Appel in 1900 [2]. The Gibbs-Appel equations for the nonholonomic system constituted of $n$ particles have been shown in a lot of specialist books $[3,4]$.

In this paper we consider a system $\mathrm{S}$ constituted of $p$ rigid bodies. The Gibbs-Appel function is defined as $[5,6]$

$$
G=\frac{1}{2} \int \vec{a}^{2} d m
$$

and the equations of motion are obtained by

$$
\frac{\partial G}{\partial \ddot{\pi}_{i}}=\Pi_{i}, \quad(i=1, \ldots, f)
$$

where $\Pi_{i}$ is the quasi-active force, $\pi_{i}$ is the quasi-coordinate, $f$ is the number of degrees of freedom of the system.

The subject of this paper is the proposition of a useful matrix form of Gibbs-Appel function of multibody system. The formulation is aimed at more automatic and clear generation of the dynamical equations of motion.

\section{THE MATRIX FORMULATION OF GIBBS-APPEL FUNCTION}

As shown in Fig. 1, which depicts a rigid body in the three-dimensional space, the global position of an arbitrary point on the body can be written as

$$
\vec{r}=\vec{r}_{A}+\vec{u}
$$

By differentiating Eq. (2.1) with respect to time one obtains the velocity of an arbitrary point on the rigid body

$$
\vec{v}=\vec{v}_{A}+\vec{\omega} \times \vec{u} .
$$

The time derivative of Eq. (2.2) gives acceleration relation as

$$
\vec{a}=\vec{a}_{A}+\vec{\alpha} \times \vec{u}+\vec{\omega} \times(\vec{\omega} \times \vec{u})
$$




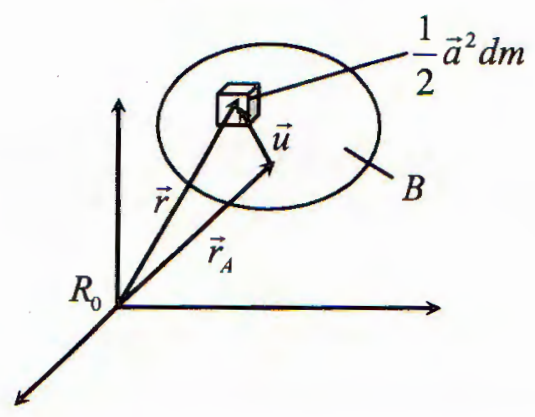

Fig. 1. Rigid body in the space

By substituting Eq. (2.3) into Eq. (2.1), one obtains the Gibbs-Appel function of the rigid body

$$
\begin{aligned}
G= & \frac{1}{2} \int_{B} \vec{a}^{2} d m=\frac{1}{2} \int_{B}\left[\vec{a}_{A}+\vec{\alpha} \times \vec{u}+\vec{\omega} \times(\vec{\omega} \times \vec{u})\right]^{2} d m \\
= & \frac{1}{2} \int_{B}\left\{\vec{a}_{A}^{2}+(\vec{\alpha} \times \vec{u})^{2}+[\vec{\omega} \times(\vec{\omega} \times \vec{u})]^{2}+2 \vec{a}_{A}(\vec{\alpha} \times \vec{u})\right. \\
& \left.+2 \vec{a}_{A}[\vec{\omega} \times(\vec{\omega} \times \vec{u})]+2(\vec{\alpha} \times \vec{u})[\vec{\omega} \times(\vec{\omega} \times \vec{u})]\right\} d m
\end{aligned}
$$

Equation (2.4) is divided into six parts, as stated in each line and calculated below

$$
\begin{aligned}
& \text { part } 1=\frac{1}{2} \int_{B} \vec{a}_{A}^{2} d m=\frac{1}{2} m \vec{a}_{A}^{2} \\
& \text { part 2 }=\frac{1}{2} \int_{B}(\vec{\alpha} \times \vec{u})^{2} d m=\frac{1}{2} \vec{\alpha}\left(\overrightarrow{\vec{I}}_{A} \vec{\alpha}\right) \\
& \operatorname{part~3}=\int_{B} \vec{a}_{A}(\vec{\alpha} \times \vec{u}) d m=\vec{a}_{A}\left(\vec{\alpha} \times \int_{B} \vec{u} d m\right)=m \vec{a}_{A}\left(\vec{\alpha} \times \vec{u}_{C}\right) \\
& \text { part } 4=\int_{B} \vec{a}_{A}[\vec{\omega} \times(\vec{\omega} \times \vec{u})] d m=\vec{a}_{A}\left[\vec{\omega} \times \int_{B}(\vec{\omega} \times \vec{u}) d m\right]=m \vec{a}_{A}\left[\vec{\omega} \times\left(\vec{\omega} \times \vec{u}_{C}\right)\right] \\
& \text { part } 5=\int_{B}(\vec{\alpha} \times \vec{u})[\vec{\omega} \times(\vec{\omega} \times \vec{u})] d m=\vec{\alpha} \vec{\omega} \times\left(\overrightarrow{I_{A}} \vec{\omega}\right) \\
& \text { part } 6=\frac{1}{2} \int_{B}[\vec{\omega} \times(\vec{\omega} \times \vec{u})]^{2} d m
\end{aligned}
$$

where $m$ is the mass of the body, $\vec{u}_{C}$ its the local center of mass position, $\vec{a}_{A}$ the acceleration of the point $A, \vec{\omega}$ the angular velocity, $\vec{\alpha}$ the angular acceleration, $\overrightarrow{\vec{I}}_{A}$ the inertia tensor of the body with respect to point $A$.

Substituting Eqs. (2.5) - (2.10) into Eq. (2.4), we get

$$
G=\frac{1}{2} m \vec{a}_{A}^{2}+\frac{1}{2} \vec{\alpha}\left(\overrightarrow{\vec{I}}_{A}^{\prime} \vec{\alpha}\right)+m \vec{a}_{A}\left(\vec{\alpha} \times \vec{u}_{C}\right)+m \vec{a}_{A}\left[\vec{\omega} \times\left(\vec{\omega} \times \vec{u}_{C}\right)\right]
$$




$$
+\vec{\alpha} \vec{\omega} \times\left(\overrightarrow{\vec{I}}_{A} \vec{\omega}\right)+\frac{1}{2} \int_{B}[\vec{\omega} \times(\vec{\omega} \times \vec{u})]^{2} d m
$$

Note that by the using of Gibbs-Appel equations, one interests only the parts of function $G$, which depend on the quasi-acceleration $\ddot{\pi}_{i}$. Hence the Eq. (2.11) can be expressed in the following form as

$$
\mathbf{G}^{-}=\mathbf{G}-\frac{1}{2} \int_{B}[\vec{\omega} \times(\vec{\omega} \times \vec{u})]^{2} d m
$$

The function

$$
G^{-}=\frac{1}{2} m \vec{a}_{A}^{2}+\frac{1}{2} \vec{\alpha}\left(\overrightarrow{\vec{I}}_{A} \vec{\alpha}\right)+m \vec{a}_{A}\left(\vec{\alpha} \times \vec{u}_{C}\right)+m \vec{a}_{A}\left[\vec{\omega} \times\left(\vec{\omega} \times \vec{u}_{C}\right)\right]+\vec{\alpha} \vec{\omega} \times\left(\overrightarrow{\vec{I}}_{A} \vec{\omega}\right)
$$

is said to be the incomplete Gibbs-Appel function.

If the mass center $C$ of a body is chosen as the point $A$ the relation (2.13) obtained reduces to

$$
G^{-}=\frac{1}{2} m \vec{a}_{C}^{2}+\frac{1}{2} \vec{\alpha}\left(\vec{I}_{C} \vec{\alpha}\right)+\vec{\alpha} \vec{\omega} \times\left(\overrightarrow{\vec{I}}_{C} \vec{\omega}\right)
$$

We now define the incomplete Gibbs-Appel function of the system of rigid bodies as follows

$$
G^{-}=\frac{1}{2} \sum_{k=1}^{p}\left[m_{k} \vec{a}_{C k}^{2}+\vec{\alpha}_{k}\left(\overrightarrow{\vec{I}}_{C k} \vec{\alpha}_{k}\right)+2 \vec{\alpha}_{k} \vec{\omega}_{k} \times\left(\overrightarrow{\vec{I}}_{C k} \vec{\omega}_{k}\right)\right]
$$

where $m_{k}(k=1,2, \ldots, p)$ is the mass of body $B_{k}, \vec{a}_{C_{k}}$ the acceleration of the mass center $C_{k}, \overrightarrow{\vec{I}}_{C_{k}}$ the inertia tensor of body $B_{k}$ with respect to the mass center $C_{k}, \vec{\alpha}_{k}$ the angular acceleration of body $B_{k}, \vec{\omega}_{k}$ the angular velocity of body $B_{k}$.

The matrix form of the incomplete Gibbs-Appel function should be expressed as

$$
G^{-}=\frac{1}{2} \sum_{k=1}^{p}\left[m_{k} \boldsymbol{a}_{C k}^{T} \boldsymbol{a}_{C k}+\boldsymbol{\alpha}_{k}^{T}\left(\mathbf{I}_{C k} \boldsymbol{\alpha}_{k}\right)+2 \boldsymbol{\alpha}_{k}^{T} \tilde{\omega}_{k}\left(\boldsymbol{I}_{C k} \boldsymbol{\omega}_{k}\right)\right]
$$

In the particular case of a rigid body rotating in three-dimensional space about a fixed point, which is chosen as the posion point $A$, according to $\mathrm{Eq}$. (2.13) the incomplete Gibbs-Appel function of the rigid body may be expressed as

$$
G^{-}=\frac{1}{2} \vec{\alpha}\left(\overrightarrow{\vec{I}}_{O} \vec{\alpha}\right)+\vec{\alpha} \vec{\omega} \times\left(\overrightarrow{\vec{I}}_{O} \vec{\omega}\right)
$$

For the general plane motion of rigid body according to Eq. (2.16) we obtaine the following matrix form of the Gibbs-Appel function

$$
G^{-}=\frac{1}{2}\left[m \boldsymbol{a}_{C}^{T} \boldsymbol{a}_{C}+\boldsymbol{\alpha}^{T}\left(\boldsymbol{I}_{C} \boldsymbol{\alpha}\right)+2 \boldsymbol{\alpha}^{T} \tilde{\omega}\left(\boldsymbol{I}_{C} \boldsymbol{\omega}\right)\right]
$$

The expressions of $\boldsymbol{a}_{C}, \boldsymbol{\omega}, \boldsymbol{\alpha}, I_{C}$ in Eq. (2.18) can be written in the following matrix form

$$
\mathbf{a}_{C}=\left[\begin{array}{l}
\ddot{x}_{C} \\
\ddot{y}_{C} \\
0
\end{array}\right], \omega=\left[\begin{array}{l}
0 \\
0 \\
\omega
\end{array}\right], \alpha=\left[\begin{array}{l}
0 \\
0 \\
\alpha
\end{array}\right], I_{C}=\left[\begin{array}{lll}
I_{1} & 0 & 0 \\
0 & I_{2} & 0 \\
0 & 0 & I_{2}
\end{array}\right]
$$

Substituting Eqs. (2.19) into Eq. (2.18) we have

$$
G^{-}=\frac{1}{2} m\left(\ddot{x}_{C}^{2}+\ddot{y}_{C}^{2}\right)+\frac{1}{2} I_{3} \alpha^{2} .
$$




\section{EXAMPLE}

Consider the motion of a sphere rolling on a inclined plane shown in Fig. 2. We assume that the sphere rolls without slipping. The sphere has the mass $m$ and the radius $a$. By using Gibbs-Appel equations, the equations of motion for the sphere can be derived as follows.

Let $q_{1}=x_{C}, q_{2}=y_{C}, q_{3}=\varphi, q_{4}=\psi, q_{5}=\theta$ denote the configuration of the sphere. The holonomic and nonholonomic constraint equations of this model are written as

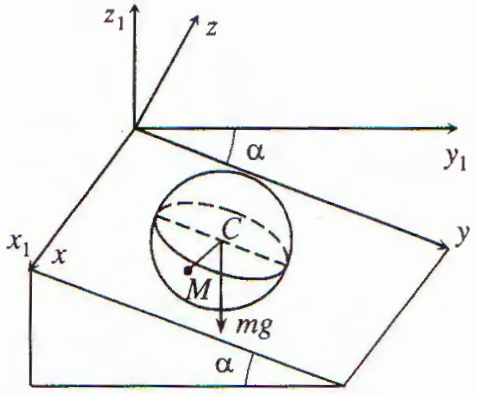

Fig. 2. A sphere rolls without slipping on a inclined plane

$$
z_{C}=a, \quad \dot{x}_{C}-a \omega_{y}=0, \dot{y}_{C}+a \omega_{x}=0
$$

From the Euler kinematics equation we have [5], [6]

$$
\begin{aligned}
& \omega_{x}=\dot{\varphi} \sin \theta \sin \psi+\dot{\theta} \cos \psi, \\
& \omega_{y}=-\dot{\varphi} \sin \theta \cos \psi+\dot{\theta} \sin \psi, \\
& \omega_{z}=\dot{\psi}+\dot{\varphi} \cos \theta
\end{aligned}
$$

Therefore one can choose the quasi-velocities as

$$
\dot{\pi}_{1}=\omega_{x}, \quad \dot{\pi}_{2}=\omega_{y}, \quad \dot{\pi}_{3}=\omega_{z}
$$

From Eqs. (3.1) and (3.3), we obtain

$$
\begin{aligned}
& \dot{q}_{1}=\dot{x}_{C}=a \dot{\pi}_{2}, \\
& \dot{q}_{2}=\dot{y}_{C}=-a \dot{\pi}_{1}, \\
& \dot{q}_{3}=\dot{\varphi}=\frac{\sin q_{4}}{\sin q_{5}} \dot{\pi}_{1}-\frac{\cos q_{4}}{\sin q_{5}} \dot{\pi}_{2}, \\
& \dot{q}_{4}=\dot{\psi}=-\dot{\pi}_{1} \sin q_{4} \cot g q_{5}+\dot{\pi}_{2} \cos q_{4} \cot g q_{5}+\dot{\pi}_{3}, \\
& \dot{q}_{5}=\dot{\theta}=\dot{\pi}_{1} \cos q_{4}+\dot{\pi}_{2} \sin q_{4}
\end{aligned}
$$

The incomplete Gibbs-Appel function reduces to

$$
G^{-}=\frac{1}{2} m \boldsymbol{a}_{C}^{T} \boldsymbol{a}_{C}+\frac{1}{2} \alpha^{T}\left(I_{C} \alpha\right)+\alpha^{T}\left(\tilde{\omega} I_{C} \omega\right)
$$

where

$$
\boldsymbol{a}_{C}=\left[\begin{array}{l}
\ddot{x}_{C} \\
\ddot{y}_{C} \\
0
\end{array}\right], \omega=\left[\begin{array}{c}
\omega_{x} \\
\omega_{y} \\
\omega_{z}
\end{array}\right], \alpha=\left[\begin{array}{c}
\dot{\omega}_{x} \\
\dot{\omega}_{y} \\
\dot{\omega}_{z}
\end{array}\right], I_{C}=\left[\begin{array}{ccc}
I & 0 & 0 \\
0 & I & 0 \\
0 & 0 & I
\end{array}\right]
$$

Substituting Eq. (3.6) into Eq. (3.5) we get

$$
G^{-}=\frac{1}{2} m\left(\ddot{x}_{C}^{2}+\ddot{y}_{C}^{2}\right)+\frac{1}{2} I\left(\dot{\omega}_{x}^{2}+\dot{\omega}_{y}^{2}+\dot{\omega}_{z}^{2}\right)
$$

According te Eqs. (3.3) and (3.4) we can also write Eq. (3.7) as

$$
G^{-}=\frac{1}{2}\left(I+m a^{2}\right) \ddot{\pi}_{1}^{2}+\frac{1}{2}\left(I+m a^{2}\right) \ddot{\pi}_{2}^{2}+\frac{1}{2} I \ddot{\pi}_{3}^{2}
$$


The virtual work of the gravity force acting on the sphere is given by

$$
\delta A=-m g a \sin \alpha \delta \pi_{1}
$$

This implies that

$$
\Pi_{1}=-m g a \sin \alpha, \quad \Pi_{2}=0, \quad \Pi_{3}=0
$$

The equations of motion for the sphere in the form (1.2) lead to

$$
\begin{aligned}
& \left(I+m a^{2}\right) \ddot{\pi}_{1}=-m g a \sin \alpha, \\
& \left(I+m a^{2}\right) \ddot{\pi}_{2}=0, \\
& I \ddot{\pi}_{3}=0
\end{aligned}
$$

These results are in agreement with the calculating results by $[3,4,6]$.

\section{CONCLUSION}

In many applications it is convenient to formulate the dynamical equations not in the generalized coordinates $q$ but in quasi-coordinates $\pi=\left[\pi_{1}, \pi_{2}, \ldots, \pi_{f}\right]^{T}$. In this paper the calculation of Gibbs-Appel function for multibody systems is considered. The advantages ensue mainly from the matrix formulation of Gibbs-Appel function for each body of the considered system. The approach and the matrix formulation seems also be well suited for development of computer algorithms.

\section{REFERENCES}

1. J. W. Gibbs, On the Fundamental Formulae of Dynamics, Am. J. Math., 2 (1897) 49-64.

2. P. Appel, Sur une forme generale des equations de dynamique, Z. Reine und Angewandte Mathematik 124 (1900) 310-319.

3. A. I. Lurie, Analytical Mechanics, Springer-Verlag, Berlin 2002.

4. J. G. Papastavridis, Analytical Mechanics, Oxford University Press, New York 2002.

5. A. A. Shabana, Computational Dynamics, 2. Edition, John Wiley \& Sons, New York 2001.

6. Nguyen Van Khang, Multibody Dynamics (in Vietnamese), Science and Technique Publishing House, Hanoi 2007.

Received October 26, 2006

\section{Vî̀ CÁC PHƯƠNG TRINHH GIBBS-APPEL CỦA HỆ NHIỀU VẬT}

Trong bài báo này trình bày việc xây dựng dạng ma trận của hàm Gibbs-Appel cho hệ nhiều vật. Dạng ma trận nêu ra phục vự thuận tiện cho việc tự động 'hoá thiết lập phương trình chuyển động của các hệ nhiều vật phi hôlônôm. Các ưu điểm của công thức nêu ra đã được khảo sát qua một thí dụ minh hoạ. 